\title{
DetR DB: a database of ionizing radiation resistance determinants
}

\author{
Alina Ryabova ${ }^{1}$, Olga Kozlova ${ }^{1}$, Azat Kadirov ${ }^{1}$, Oleg Gusev ${ }^{1}$, Elena Shagimardanova 1,* \\ 1 Kazan Federal University, Kazan, Russia; rjuka@mail.ru \\ * Correspondence: rjuka@mail.ru;
}

\begin{abstract}
Nuclear pollution is an urgent environmental issue as a consequence of rapid industrialization and nuclear accidents in the past. Remediation of nuclear polluted sites using microbial vital activity (bioremediation) is a promising approach to recover contaminated areas in an environmentally friendly and cost-saving way. At the same time, the number of known bacterial and archaeal species able to withstand extremely high doses of ionizing radiation is steadily growing every year, together with growing knowledge about mechanisms of radioresistance. This opens up new opportunities for developing new biotechnological solutions. However, these data are often not systemized and it can be difficult to access. Here, we present the Determinants of Radioresistance Database, or DetR DB (http://extremebiolab.com/detr-db/), gathering a comprehensive catalog of radioresistant microbes and their molecular and genetic determinants of enhanced ionizing radiation tolerance. The database provides search tools including taxonomy, common gene name, and BLAST. DetR DB will be a useful tool for research community by facilitating the extraction of the necessary information to help further analysis of radiation-resistant mechanisms.
\end{abstract}

Keywords: ionizing radiation; radiation resistance; genetic mechanisms; microorganisms

\section{Introduction}

Ionizing radiation (IR) is widely presented in daily lives of all organisms, originating from natural and manmade sources. Environmental IR or natural background radiation is largely the cosmic rays from deep in space and the naturally radioactive elements that are relatively abundant in rocks and soils and so can be found in water, vegetation and foods. Background radiation levels vary geographically due to geological differences, although mostly at low levels. Artificial IR comes from human-made sources ranging from the nuclear fuel cycle and industry to medical application for diagnosis and treatment $[1,2]$.

It is worth mentioning separately the IR exposure from radioactive pollution which may be extremely hazardous. It occurs when there is a presence or depositions of radioactive substances, especially as a result of nuclear accidents, acts of military aggression or while conducting nuclear tests [3]. Two nuclear fallouts in Hiroshima and Nagasaki (1945) in the Second World War, and a number of the most mournful nuclear power plant accidents like Three Mile Island accident (1979), Chernobyl (1986) and the Fukushima Daiichi (2011) nuclear disasters left many instantly dead and caused severe damage to the environment for many more years by the radiation released [4]. Radioactive contamination is a serious threat to almost all living forms since the pollution maintains its radioactive properties for decades to millennia [2].

Radiation-induced damage is determined by parameters including the type and dosage of ionizing radiation rays, length of exposure, and, importantly, the characteristics of the exposed individual [5]. An exceptional ability to withstand the lethal effects of ionizing radiation is widespread among organisms that inhabit extreme environments. These organisms, known as extremophiles, are a largely unexplored group that survives in habitats that are hostile or even 
deadly for other life forms [6]. Extremophiles are found in all domains of life, but the vast majority of them belong to Bacteria and Archaea species. Such extremophilic microorganisms are highly adapted to thrive in natural conditions, encompassing broad ranges of temperature, salinity, $\mathrm{pH}$, water, and nutrient limitation, which constitute the norm they are able to metabolically and biochemically operate. In addition to natural habitats that can be defined as "extreme" from anthropocentric criteria, many independent scientific works have demonstrated life to be present in artificial environments with adverse factors for beings, even high levels of IR. Thus, some extremophilic bacterial and archaeal species have been isolated from high-level radioactive waste sites at Savannah River in South Carolina and at Hanford in Washington [7, 8], spent nuclear fuel storage pools at Sellafield, UK [9] and Madras Atomic Power Station, Kalpakkam [10], German salt dome Gorleben [11], from the Chernobyl Nuclear Power Plant buildings [12, 13, 14]. According to the most widely accepted theory, the origin of cross-resistance to ionizing radiation and other types of stress may be related to overlap between mechanisms of resistance to IR and other stressors [15]. However, radioresistance is not limited only to extremophiles, but can occur among common microbial species, as it was found in river sediment soils exposed to radioactive contamination from Fukushima [16], or it can be cultivated in laboratory from radiosensitive variants [17].

Radiation resistant microorganisms possess a huge potential for biotechnological applications in the field of bioremediation of radioactive waste in an eco-friendly and cost-saving way. To date, the most developed bioremediation method based on the microbial usage employs the widely known radiation-resistant bacterium Deinococcus radiodurans [18], although researches are underway on the possible use of other microorganisms for these purposes [19, 20, 21, 22, 23]. On the other hand, in addition to the use of such microbes in the disposal of radioactive waste, it is unclear how radioresistant microbes affect the structural materials of nuclear waste storage facilities and the environment of nuclear reactors, and whether they can lead to their biodegradation. Anyhow, resistance to high doses of ionizing radiation is obviously a "hot" topic, but despite it, there is not much organized up-to-date information about radioresistant organisms and their mechanisms of radioresistance.

In order to combine and systematize rapidly accumulating knowledge about those organisms that are able to thrive in conditions of high levels of ionizing radiation, we designed the Determinants of Radioresistance Database (DetR DB, http://extremebiolab.com/detr-db/). This is an open-access online tool based on peer-reviewed articles of radioresistant microorganisms with emphasis on their known molecular and genetic benefits that determine enhanced ionizing radiation tolerance. The current version of the database contains 63 species of Bacteria and Archaea domains and a description of about four thousand genes. Users can search DetR DB by several fields, including taxonomy or BLASTn/BLASTp. We hope that DetR DB can make it more comfortable for researchers to get the necessary data and help in the early development of effective bioremediation methods.

\section{Database design}

The DetR DB compiles both manually curated information and automatically generated data that is regularly managed and updated. Firstly, we analyzed the open-access published literature available in the NCBI database, covering all reports of isolation and identification of radioresistant microorganisms and giving preference to researches with specified D10 value (D10 threshold is the absorbed radiation dose required to inactivate $90 \%$ of a viable population). At the time of writing, the database includes 55 species of Bacteria domain and 8 species of Archaea domain (Figure 1). The same procedure was used to search for genes, proteins or pathways involved in the formation and functioning of mechanisms that determine the resistance to ionizing radiation. As with the selection process of most microbial species, we focused on those studies where the role of the specific determinant was confirmed experimentally, thus the database currently contains 119 genes of interest. Each strain of selected microorganism was matched with the corresponding UniProt ID number that was used to get and bind diverse data on species and genes. Taxonomy of 
radioresistant microbes was obtained from NCBI database. The metadata on genes and proteins, including amino acid sequences, were gathered from UniProt. Nucleotide sequences and gene ontology (GO) annotation were downloaded from KEGG database. The final database was transferred to our web server (http://extremebiolab.com/detr-db/). This software was written in C\# with .NET Framework 4 platform usage. Windows Presentation Foundation (WPF) was used to build the user interface.

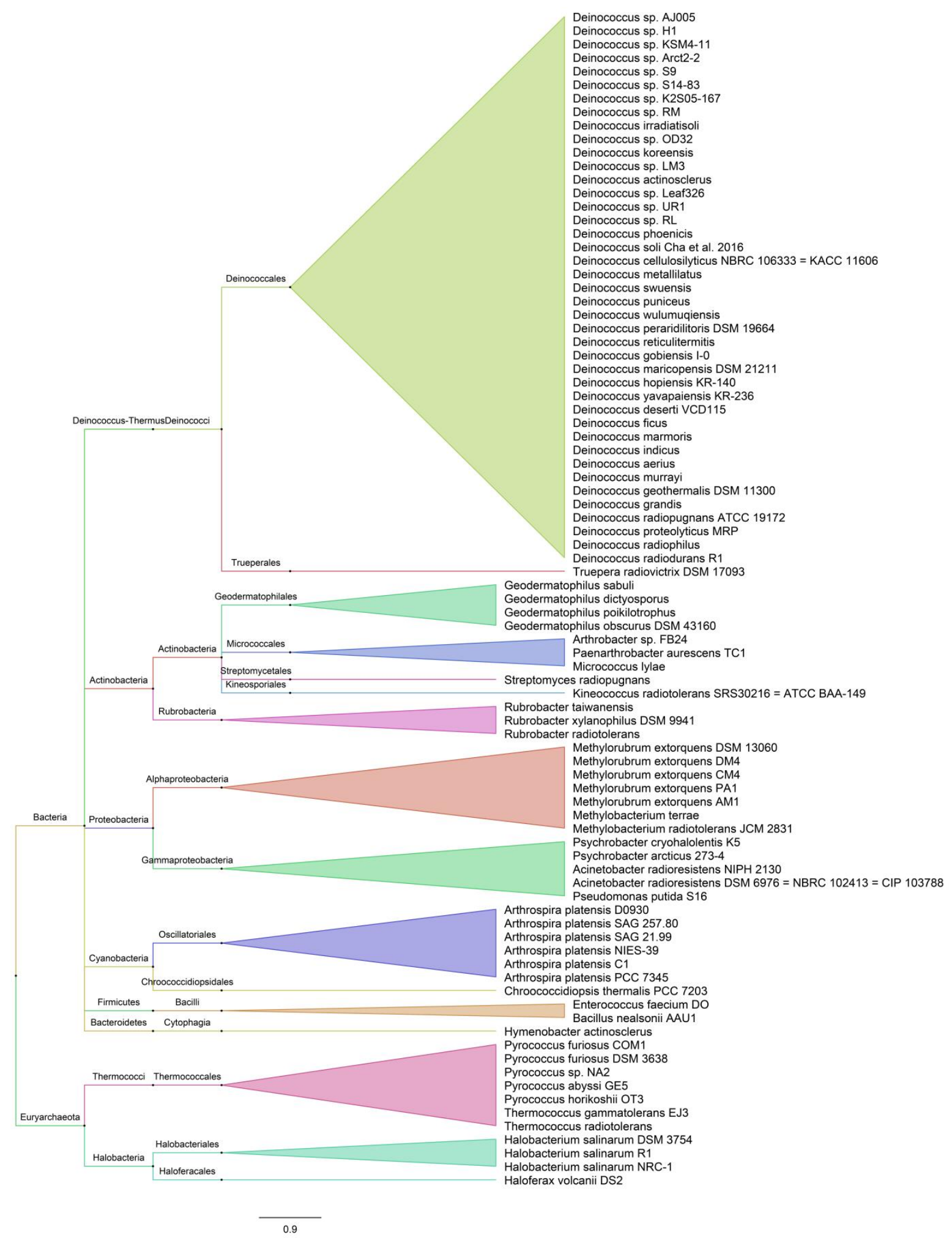

Figure 1. Phylogenetic tree containing all microbial species in the DetR DB. 


\section{Database filling and options}

The DetR DB is exclusively focused on relevant information about genetic and molecular determinants of enhanced radioresistance in a species-specific manner. Therefore, the content of the database is organized in three sections that contain BLAST search, taxonomy of microorganisms and genes annotation (Figure 2).

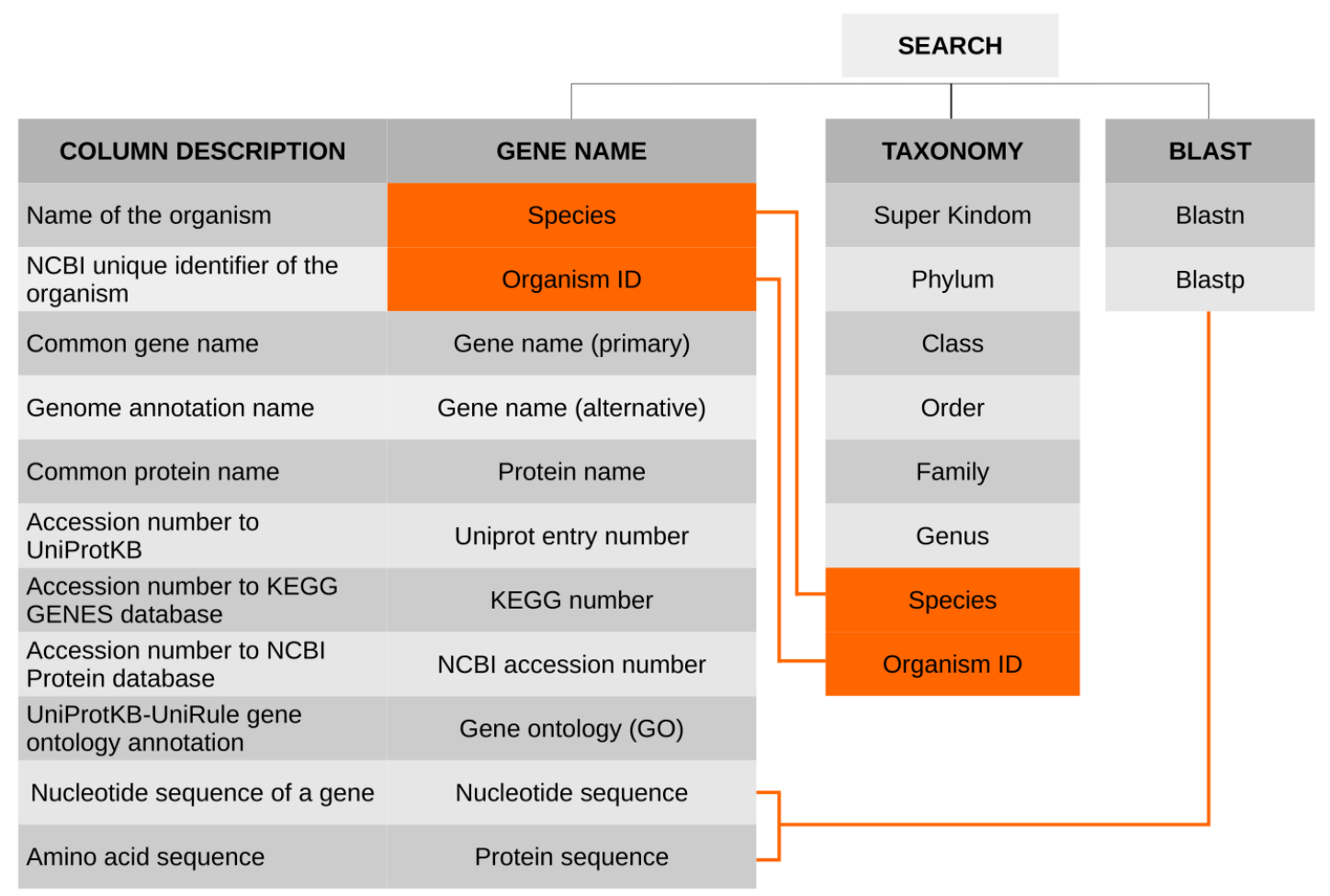

Figure 1. Phylogenetic tree containing all microbial species in the DetR DB.

The last two sections are interconnected via specific links. The taxonomy section provides a description of the taxonomic lineage of microorganism, including the main nomenclature ranks from domain to strain, as well as the organism id number (Uniprot). The gene annotation section contains metadata that defines the species or strain with its organism id number and its determinant: primary gene name, alternative gene name, protein name, Uniprot entry number, KEGG number, NCBI accession number, gene ontology (GO), nucleotide sequence and protein sequence. There are two search modes for these sections: by taxonomy and by gene name, respectively. The taxonomy search can be carried out by entering any taxonomic rank, strain name or organism id. The gene name search, as the title suggests, allows making a query through the common gene name as a keyword. The database can be searched with sequence-based queries by the standard BLAST tool that will compare a user-submitted sequence against nucleotide or amino acid sequence records in DetR DB (Figure 3). 


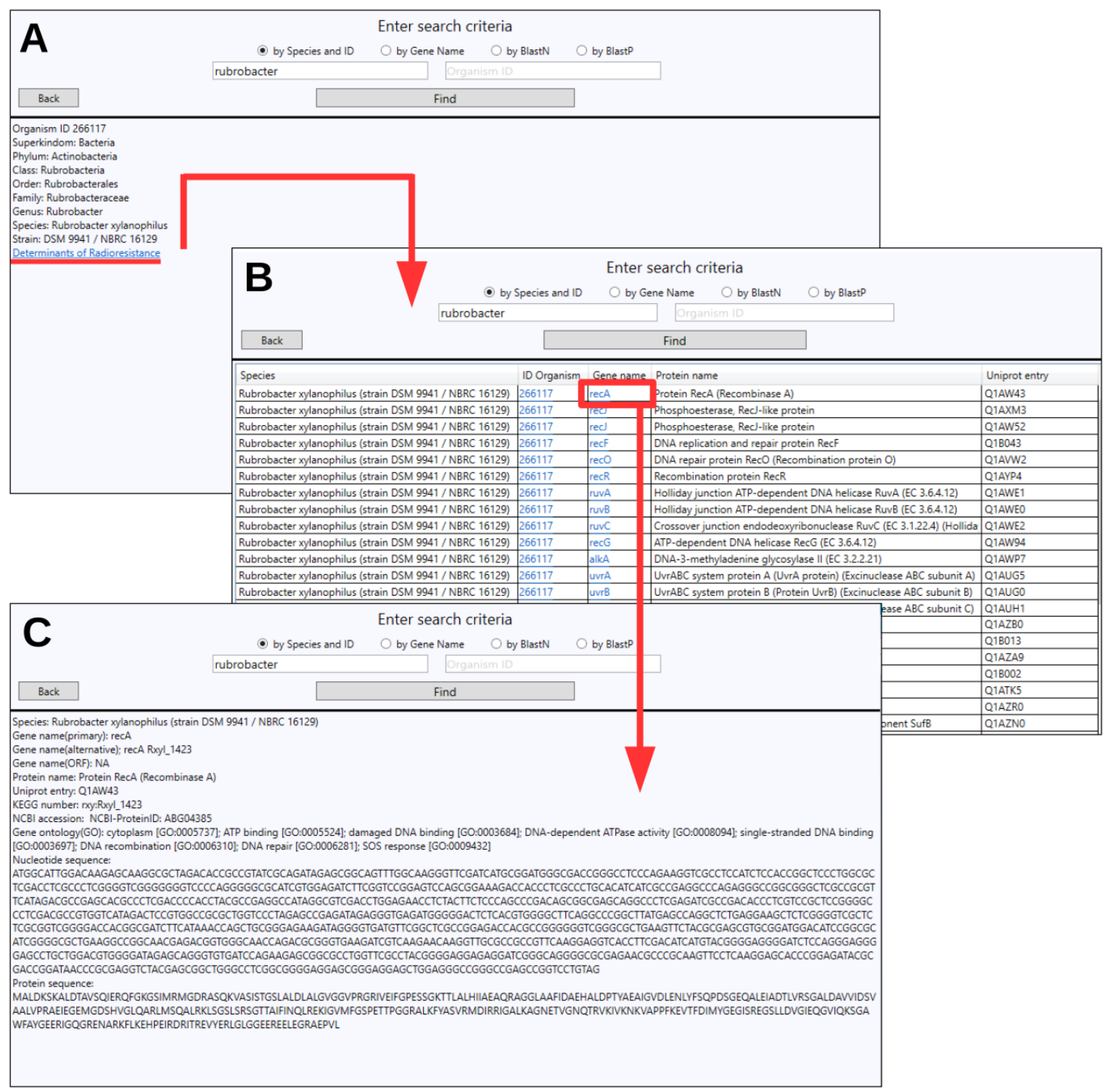

Figure 3. The example of search and navigation in the DetR DB. (a) The organism description page provides organism ID, taxonomy and the link to the list of all determinants of ionizing radiation resistance for this species that are contained in the database; (b) The list of genes related to enhanced radioresistance of particular microorganisms, including common gene name, protein name and Uniprot entry number; (c) From the list of radioresistance determinants, the user can select a gene of interest and go to the page with its detailed description.

\section{Future plans}

DetR DB will continue to grow with inclusion of a new published data and adding more details to existing ones. We intend to incorporate more radioresistant bacterial and archaeal species from studies that we may have overlooked, as well as from those that are still to come in the future. Expanding the database with eukaryotic organisms also could be of great interest and practical use, since a large number of extremely ionizing radiation-resistant organisms are known among fungi species or even several invertebrates. Besides, DetR DB continues to make improvements to the operational stability and user interface convenience. The visual component is planned to be given a more attractive appearance, while the interface will be provided with an advanced navigation features. We continue to work on providing high quality and up-to-date data on radioresistant organisms and their mechanisms of endurance to ionizing radiation. Thereby, the DetR DB could become an efficient platform for evolutionary studies or developing synthetic biology applications that may eventually lead to creating of environmentally friendly bioremediation technologies. 
Author Contributions: Conceptualization, O.G. and E.Sh; methodology, O.K. and A.R.; software, A.K.; validation, E.Sh and A.R.; writing-original draft preparation, A.R.; writing-review and editing, A.R., O.G., E.Sh., O.K.; visualization A.R.; project administration, E.Sh.; funding acquisition, E.Sh. All authors have read and agreed to the published version of the manuscript.

Funding: This research was funded by the Federal Target Program "Research and Development in Priority Areas of Development of the Russian Scientific and Technological Complex for 2014-2020" No RFMEFI61419X0004.

Acknowledgments: The authors would like to thank Alexander Cherkasov and Nikita Molostov for their contribution to the database construction.

Conflicts of Interest: The authors declare no conflict of interest.

\section{References}

1. https://www.who.int/ (accessed on 18.08.2020).

2. Koga, N. Technical overview of key agricultural events since the Fukushima Daiichi nuclear power plant accident in 2011. In Strategies and practices in remediation of radioactive contamination in agriculture, Proceedings of the Technical Workshop Vienna, Austria, 17-18 October 2016 Name of the Conference, Location of Conference, Country, Date of Conference; IAEA: Vienna, Austria, 2020.

3. Valentin J. International Commission on Radiological Protection. Protecting people against radiation exposure in the event of a radiological attack. In A report of The International Commission on Radiological Protection. Ann ICRP. 2005; Volume 35, pp 1-110. doi: 10.1016/j.icrp.2005.01.001.

4. Coeytaux, K.; Bey, E.; Christensen, D.; Glassman, E.S.; Murdock, B.; Doucet, C.; Reported radiation overexposure accidents worldwide, 1980-2013: a systematic review. PLoS One. 2015 Volume 10: e0118709. doi: 10.1371/journal.pone.0118709.

5. Reisz, J.A.; Bansal, N.; Qian, J.; Zhao, W.; Furdui, CM.; Effects of ionizing radiation on biological molecules--mechanisms of damage and emerging methods of detection. Antioxid Redox Signal. 2014, Volume 21, 260-292. doi:10.1089/ars.2013.5489.

6. Rampelotto, P.H.; Extremophiles and extreme environments. Life. 2013, Volume 3, 482-485. doi: 10.3390/life3030482.

7. Fredrickson, J.K.; Zachara, J.M.; Balkwill, D.L.; Kennedy, D.; Shu-mei, W.L.; Kostandarithes, H.M.; Daly, M.J.; Romine, M.F.; Brockman, F.J. Geomicrobiology of high-level nuclear waste-contaminated vadose sediments at the Hanford Site, Washington State. Appl Environ Microb. 2004, Volume 70, 4230-4241. doi: 10.1128/aem.70.7.4230-4241.2004

8. Bagwell, C.E.; Bhat, S.; Hawkins, G.M.; Smith, B.W.; Biswas, T.; Hoover, T.R.; Saunders, E.; Han, C.S.; Tsodikov, O.V.; Shimkets, L.J. Survival in nuclear waste, extreme resistance, and potential applications gleaned from the genome sequence of Kineococcus radiotolerans SRS30216. PLoS One. 2008, Volume 3: e3878. doi: 10.1371/journal.pone.0003878.

9. MeGraw, V.E.; Brown, A.R.; Boothman, C.; Goodacre, R.; Morris, K.; Sigee, D.; Anderson, L.; Lloyd, J.R. A novel adaptation mechanism underpinning algal colonization of a nuclear fuel storage pond. mBio. 2018, Volume 9: e02395-17. doi: 10.1128/mBio.02395-17.

10. Karley, D.; Shukla, S.K.; Rao, T.S. Microbiota of spent nuclear fuel pool water with emphasis on their biofilm forming ability on stainless steel (SS-304L). Journal of biosciences. 2019, Volume 44, 108. doi: 10.1007/s12038-019-9937-8.

11. Bader, M.; Müller, K.; Foerstendorf, H.; Schmidt, M.; Simmons, K.; Swanson, J.S.; Reed, D.T.;, Stumpf, T.; Cherkouk, A. Comparative analysis of uranium bioassociation with halophilic bacteria and archaea. PLoS One. 2018, Volume 13: e0190953. doi: 10.1371/journal.pone.0190953.

12. Zavilgelsky, G.B.; Abilev, S.K.; Sukhodolets, V.V.; Ahmad, S,I. Isolation and analysis of UV and radio-resistant bacteria from Chernobyl. Journal of Photochemistry and photobiology. B, Biology. 1998, Volume 43, 152-157. doi: 10.1016/s1011-1344(98)00099-2.

13. Romanovskaia, V.A.; Rokitko, P.V.; Mikheev, A.N.; Gushcha, N,I; Malashenko, I.; Chernaia, N.A. The effect of gamma-radiation and desiccation on the viability of the soil bacteria isolated from the alienated zone around the Chernobyl Nuclear Power Plant. Microbiology (Moscow). 2002, Volume 71, 705-712. 
14. Egorova, A.S.; Gessler, N.N.; Ryazanova, L.P.; Kulakovskaya, T.V.; Belozerskaya, T.A. Stress Resistance Mechanisms in the Indicator Fungi from Highly Radioactive Chernobyl Zone Sites. Microbiology (Moscow). 2015, Volume 84, 184-191.

15. Shuryak, I. Review of microbial resistance to chronic ionizing radiation exposure under environmental conditions. J Environ Radioact. 2019, Volume 196, 50-63. doi: 10.1016/j.jenvrad.2018.10.012.

16. Yamanouchi, K.; Tsujiguchi, T.; Shiroma, Y.; Suzuki, T.; Tamakuma, Y.; Yamaguchi, M.; Sakamoto, Y.; Hegedűs, M.; Iwaoka, K.; Hosoda, M.; Kashiwakura, I.; Miura, T.; Tokonami, S. Comparison of bacterial flora in river sediments from Fukushima and Aomori prefectures by 16s rDNA sequence analysis. Radiation Protection Dosimetry. 2019, Volume 184, 504-509. doi:10.1093/rpd/ncz114.

17. Harris, D.R.; Pollock, S.V.; Wood, E.A.; Goiffon, R.J.; Klingele, A.J.; Cabot, E.L.; Schackwitz, W.; Martin, J.; Eggington, J.; Durfee, T.J.; Middle, C.M.; Norton, J.E.; Popelars, M.C.; Li, H.; Klugman, S.A.; Hamilton, L.L.; Bane, L.B.; Pennacchio, L.A.; Albert, T.J.; Perna, N.T.; Cox, M.M.; Battista, J.R. Directed evolution of ionizing radiation resistance in Escherichia coli. J. Bacteriol. 2009, Volume 191, 5240-5252. doi: 10.1128/jb.00502-09

18. Brim, H.; Osborne, J.P.; Kostandarithes, H.M.; Fredrickson, J.K.; Wackett, L.P.; Daly, M.J.; Deinococcus radiodurans engineered for complete toluene degradation facilitates $\mathrm{Cr}(\mathrm{VI})$ reduction. Microbiology. 2006, Volume 152, 2469-2477. doi: 10.1099/mic.0.29009-0.

19. Stephen, J.R.; Macnaughton, S.J. Developments in terrestrial bacterial remediation of metals. Curr Opin Biotechnol. 1999, Volume 10, 230-233. doi: 10.1016/s0958-1669(99)80040-8.

20. Das, N. Remediation of Radionuclide Pollutants through Biosorption - an Overview. Clean Soil Air Water. 2012, Volume 40, 16-23. doi:10.1002/clen.201000522.

21. Prakash, D.; Gabani, P.; Chandel, A.K.; Ronen, Z.; Singh, O.V. Bioremediation: a genuine technology to remediate radionuclides from the environment. Microb Biotechnol. 2013, Volume 6, 349-360. doi: 10.1111/1751-7915.12059.

22. Malla, M.A.; Dubey, A.; Yadav, S.; Kumar, A.; Hashem, A.; Abd Allah, E.F. Understanding and designing the strategies for the microbe-mediated remediation of environmental contaminants using omics approaches. Front Microbiol, 2018, Volume 9: 1132. doi: 10.3389/fmicb.2018.01132.

23. Shukla, A.; Parmar, P.; Saraf, M.; Patel B. Isolation and screening of bacteria from radionuclide containing soil for bioremediation of contaminated sites. Environmental Sustainability, 2019, Volume 2, 255-264. doi: 10.1007/s42398-019-00068-y. 